\title{
Nanoprobe spectroscopy of capillary forces and its application for a real surface diagnostics
}

\author{
A.A. Efremov, P.M. Lytvyn*, A.O. Anishchenko, O.M. Dyachyns'ka, T.A. Aleksyeyeva', I.V. Prokopenko \\ V. Lashkaryov Institute for Semiconductor Physics, NAS of Ukraine \\ 41, prospect Nauky, 03028 Kyiv, Ukraine, \\ *Corresponding author: phone/fax: +380445255940,e-mail: plyt@microscopy.org.ua \\ ${ }^{l}$ G. Kurdyumov Institute for Metal Physics, NAS of Ukraine
}

\begin{abstract}
The paper presents an overview and analysis of the most reliable and at the same time rather simple theoretical models describing liquid nanomeniscus geometry and forces occurring between atomic force microscope (AFM) probe and a real surface. There are experimental results in capillary bridge force rupture measured in air, and interaction force under water buffer obtained over surfaces of different nature. It is shown that the theoretical models quite adequately describe the processes observed experimentally and, in particular, bridge ruptures dynamics at the vertical probe withdraw for different speeds. Discussed here are some methodological peculiarities and nanocapillary force spectroscopy diagnostic capabilities for surface energy mapping, prospects of using of a liquid nanomeniscus in local nanochemistry, nanolithography and nano-electrochemistry of a surface.
\end{abstract}

Keywords: capillary nanobridges, water meniscus, force-distance curves, contact angle, wettability, adhesion, atomic force microscopy.

Manuscript received 23.12.09; accepted for publication 25.03.10; published online 30.04.10.

\section{Introduction}

Moisture condensation, formation of liquid films and capillary bridges between nanoparticles play an important role in various technological, medical and biological processes affecting the adhesive and lyophilic/lyophobic surface properties. For example, it is largely evident in the interaction between the particles of granular materials [1], adhesion, friction [2, 3], corrosion, micro- and nanomechanical device operation, adhesion of cells and microorganisms to surface, which determines the toxic [4], antiseptic, and biocompatible implant properties [5-7], etc. It is important that these phenomena impact on operation of nonvacuum nanoprobe diagnostic and technological devices such as atomic force, capacitive and resistive microscopes, devices for nanomanipulations and nanopreparations, nanoprobe lithography, etc. [8-12].

Controlled capillary interaction at the nanoscale reveals a number of new potential diagnostic and technological capabilities. The most flexible and high informative devices in this branch are the atomic force microscopes that can operate in different environments, providing simultaneously surface topography and force spectroscopy data with subnanometer and picoNewton resolution, respectively. Thus, under controlled humidity, quantitative measurements of capillary forces between atomic force microscope probe and nanoobject make it possible to obtain data on local wetting, and consequently the surface energy with a spatial local resolution in order of the diameter of capillary bridge $(10-20 \mathrm{~nm})$ [13]. It is possible to gain control on field induced electrochemical reactions in nanovolume [14], monitor the chemical reaction course by changing the nanodrops properties, make deep-pen nanolitography [15], etc.

First, the capillary forces were described in details by Haines [16] and Fisher [17]. Over the past decade, considerable progress was achieved in measurements and theoretical description of nanoparticle capillary interactions with flat surfaces and each other under conditions of low roughness, homogeneity and inertness. In this work, the authors make an attempt to develop systematic theoretical and experimental approaches for utilization of capillary forces as a separate instrument for real surface diagnostics by means of atomic force spectroscopy. There is a comparative analysis of the most successful theoretical approaches and experiments, 
which illustrates several effects determining the nanocapillary interactions with real surfaces. It was experimentally illustrated that the capillary forces on real surfaces have more complex behavior depending on surface roughness, geometry of interacting bodies, lyophility, dynamics of capillary bridge geometry changes and others. Behavior of these capillary forces is poorly described in the simplest theories. Besides, complexity is presented in effects of heterogeneous surfaces, especially the solid liquid interface, and interaction between two surfaces in a liquid medium. Nevertheless, these problems can be solved using more advanced numerical models adequately constructed on the basis of simple theories considered in this paper. In addition, the important role is played by a correct strategy of the experiment using all force spectroscopy possibilities.

\section{Experimental procedure}

The measurements of nanocapillary forces were carried out by AFM using the force spectroscopy method. For these measurements, the dependences of interaction force value on probe-surface distance are registered (Fig. 1). Before force measurements, the surface area under investigation is scanned by AFM in a contact operation mode to obtain image of relief features. Then this topography mapping is stopped and AFM probe is withdrawn for a given height above the surface at the selected point. Further probe provides only a vertical movement approaching to the surface until it reaches the given value of the repulsion force. This short-range force occurs when the electronic orbitals of the probe and surface begin to overlap each other (Born repulsion). After reaching a maximum force of contact interaction, the probe is withdrawn from the surface. During the vertical displacement, the resultant surface force acts on the probe. This resultant force is balanced by the cantilever probe deformation force (elastic deflection). Thus, registering the value of an elastic AFM cantilever probe deflection it is possible to measure the interaction forces from pico- to microNewton (depending on the cantilever probe stiffness) with sub-angstrom control distance resolution. These dependences are called as force-versus-distance curves or just force-curves.

A schematic view of a typical force curves is shown in Figure $1 \mathrm{~b}$. In the position $\mathrm{I}$, the probe is far from surface, and the interaction between them is missing. In the position II, the probe is approached to the surface so close (movement along a curve is indicated by arrow), that jumps to contact with the surface due to action of van der Waals attraction forces. Thus, if the measurement takes place in air, the layer of liquid condensed on the surface can play a significant role. In the position III, the probe reaches the given maximum repulsion force and is withdrawn from the surface. Hysteresis occurs due to adhesive forces of attraction. The most significant among attraction forces is the capillary force caused by liquid bridge formation
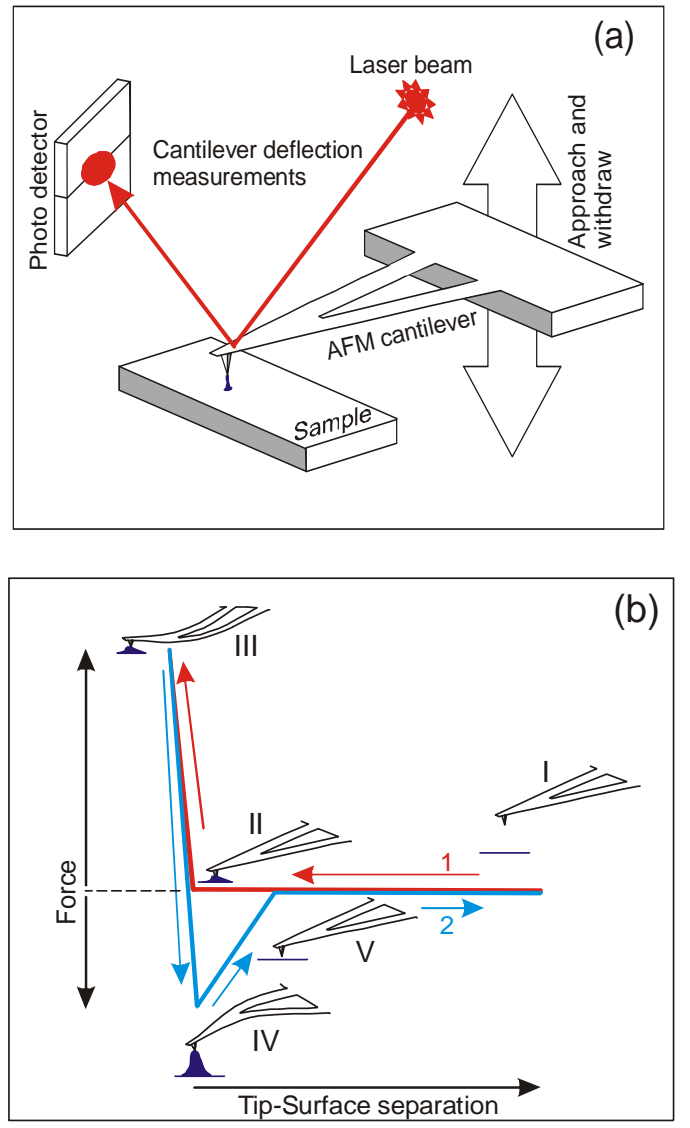

Fig. 1. Scheme of measurements (a) and schematic view of force curves when measured in air (b): approaching curve (1), withdrawing curve (2).

between the probe and surface. In the position IV, the elastic deformation force of deflected cantilever exceeds the capillary force and breaks the liquid bridge. In the position $\mathrm{V}$, the AFM probe cantilever returns to the equilibrium state. To measure the value of adhesion without affects of a liquid bridge, condensed from air, it is enough to perform measurements in a liquid medium (where liquid-air boundary is absent).

Our measurements of the force curves on various surfaces were carried out using a scanning probe microscope NanoScope IIIa both in air (at $20^{\circ} \mathrm{C}$ and relative humidity of $30 \%$ ), and in liquid buffer (distilled water). The speed of probe vertical movement was varied between 20 and $2000 \mathrm{~nm} / \mathrm{s}$. The silicon nitride V-shaped DNP-20 contact mode probes (Veeco Inc.) were used for measurements. The averaged tip apex radius was obout $30 \mathrm{~nm}$, cantilever stiffness was $0.06 \mathrm{~N} / \mathrm{m}$. The tip shape was controlled before and after measurements by using the "blind reconstruction" algorithm [18] with the TGT-1 test grating from NT-MDT. The cantilever stiffness value was checked by analyzing the spectrum of its thermal noise [19]. Typical tip shape is shown in Fig. 2a. It looks like a quadrangular pyramid. The tip apex has an elliptical shape of the curvature radius within 26 to $52 \mathrm{~nm}$. Cross-section with the largest radius of the tip is shown in Fig. 2b. It is clear 

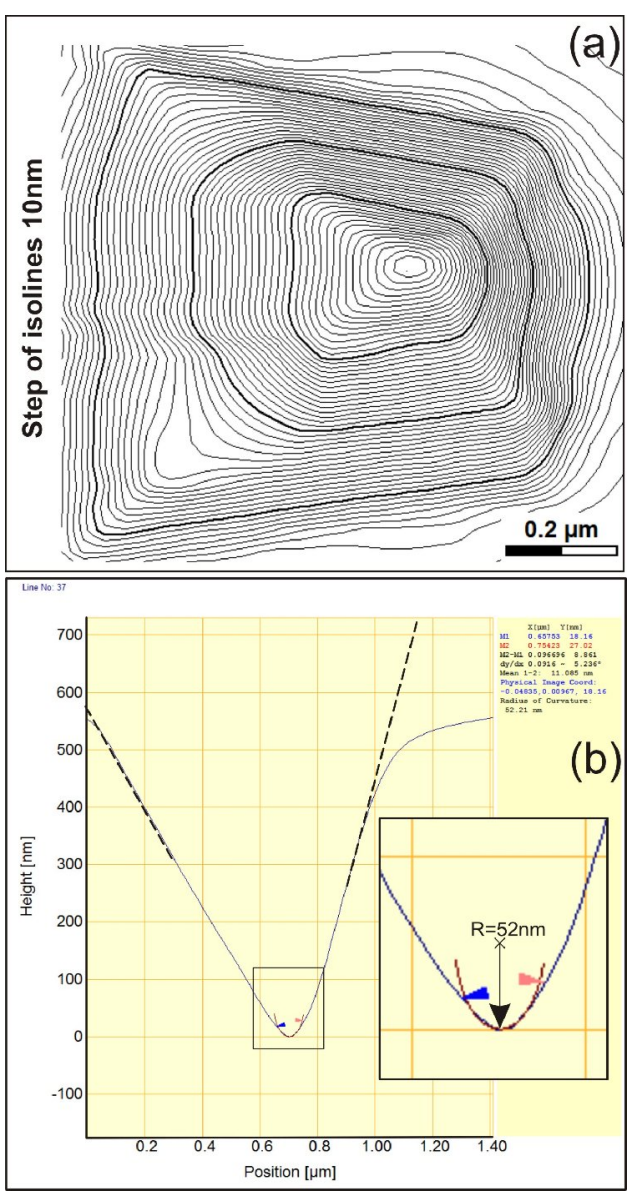

Fig. 2. The reconstructed shape of a real AFM tip apex (a) and cross-section (scale $\mathrm{X}: \mathrm{Y}=1: 2$ ) which shows the largest tip radius (b). Insert shows the approximation of tip profile by a semi-circle of the $52-\mathrm{nm}$ radius.

seen from the insert of this figure that the tip apex is well approximated by a circle of the 52-nm radius up to the 25-nm distance. This fact enables us to use the model of a spherical probe in calculations. To avoid a possible surface modification and to minimize the impact of relief, series of force curves were measured with the 100 -nm surface offset covering the area of $1 \times 1 \mu \mathrm{m}^{2}$ (there are 100 curves in one chosen area element).

\section{The physical model of AFM tip interaction with a flat surface in the case of water meniscus}

\section{Preliminary remarks. The geometry of the meniscus.}

The tip apex contacts with surface forming a narrow slit of a variable width within the contact area. If the contacting surfaces are lyophilic (hydrophilic), in relation to the surrounding vapor, some vapor will condense in the contact vicinity and form a meniscus. This phenomenon is similar to condensation of a bulk liquid in the cracks and pores on real surface.
The meniscus is often approximately modeled by a cylinder. As depicted in Fig. 3 in the more detailed analytical models, the meniscus is considered as a rotation body formed by the arc of radius $r$. The arch curvature center is separated from the rotation axis (the axis OY in Fig. 3a) at a distance $d=r+a$. This figure called as a pendular ring belongs to the family of toroids. It is the torus internal part (its hole) bounded with two cutting planes above and below. Thus, $a$ is called as the azimuth radius, and $r$ is called as the meridional radius of a liquid bridge.

The meniscus geometry forming in the so-called circular or toroidal approximation is shown in Fig. $3 \mathrm{~b}$. As shown, $a, b$, and $c$ are the meniscus radii at the neck, at the probe and at the sample plane, respectively (Fig. 3). Let $\Phi$ and $\theta$ be wetting angles for the material surface and the probe, respectively, and $\varphi$ is the socalled filling angle correlating with the environment humidity.

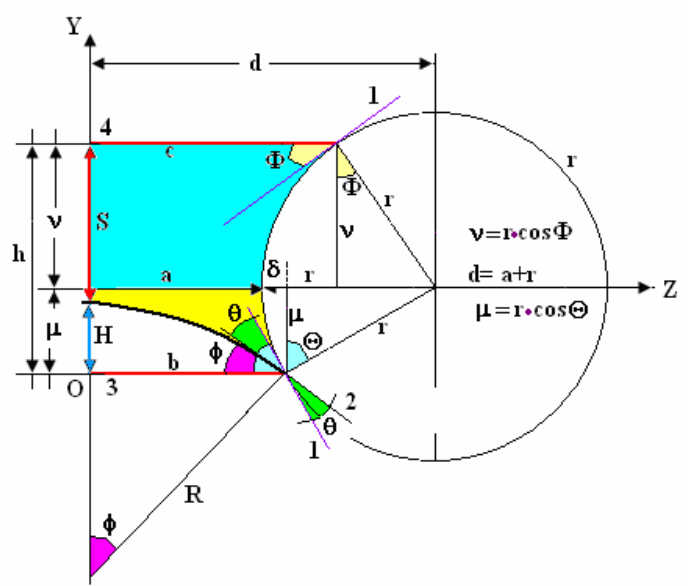

a)

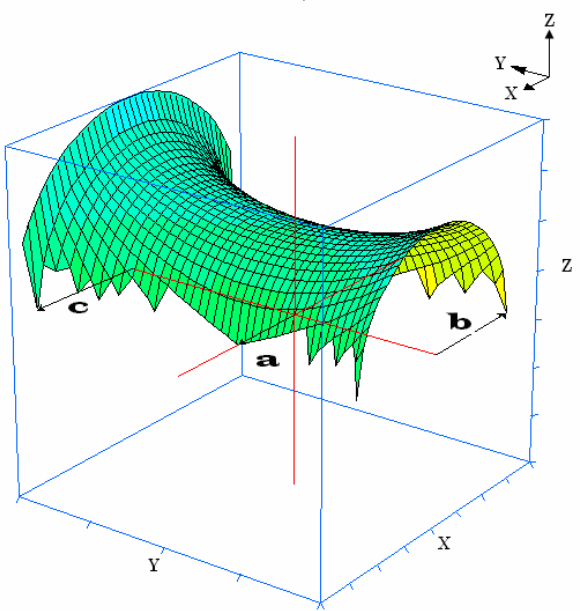

b)

Fig. 3. Three-dimensional meniscus image (b) and its cross section in the plane $\mathrm{YZ} \mathrm{(a):} a$ is a neck radius, $r$ is a side surface curvature radius, $b$ and $c$ are bases radii, 1 is a tangent to the meniscus profile at the 3 -phase contact point, 2 is a tangential to the tip surface, 3 is a tip wetting plane, 4 is a sample plane. 
Then, the depth of immersion $H=R(1-\cos \varphi)$, tip wetted area radius $b=R \sin \varphi$ determine how deeply the probe is dipped into the liquid in contact.

Hence, the angle between the meniscus and the horizon at the 3-phase contact point on the sphere is $\Theta=\varphi+\theta$.

The distance from the meniscus center to the wetting appropriate plane is determined by the corresponding 3-phase contact angle $\{\Theta, \Phi\}$ "meniscusplane". In the toroidal approximation, the meniscus surface area and volume are evaluated analytically using the formulas (1) and (2):

$S=2 \pi^{2} d \cdot r-2 \pi \cdot r^{2}(\cos \Theta+\cos \Phi)-$

$-2 \pi d \cdot r(\Theta+\Phi)$,

where $d=r+a$.

The exact expression for the liquid volume in the meniscus is (as a body of rotation):

$V=-\frac{\pi r^{3}}{3}\left(\cos ^{3} \Theta+\cos ^{3} \Phi\right)+\cos \Theta \cdot\left[\pi r\left(d^{2}+r^{2}\right)-\right.$

$\left.-\pi d r^{2} \sin \Theta\right]+\cos \Phi \cdot\left[\pi r\left(d^{2}+r^{2}\right)-\pi d r^{2} \sin \Phi\right]-$

$-\pi d r^{2}(\Theta+\Phi-\pi)-$

$-\frac{\pi R^{3}}{3}\left(2-3 \cos \varphi+\cos ^{3} \varphi\right), \Theta=\theta+\varphi$.

Various formulas for the pendular ring volume and surface area obtained by different authors are systematized in the review [20].

However, in practice the simplified models are used when the form subtleties are not recorded and the pendular ring is approximated by a cylinder. In equilibrium vapor-liquid, the average curvature of the meniscus concave side is determined by the (negative) radius of Kelvin [21]:

$\left(\frac{1}{a}-\frac{1}{r}\right)^{-1}=r_{K}=\frac{\gamma_{L} \Omega}{k_{B} T \ln \left(p / p_{s}\right)}=\frac{\lambda_{K}}{\ln \left(p / p_{s}\right)}$,

where $\Omega$ is the molecular volume, $\gamma_{L}$-surface tension (for water $-72.8 \mathrm{mN} / \mathrm{m}$ ), $p / p_{S}$ - relative humidity, $\lambda_{K}-$ the so-called Kelvin length, corresponding to $r_{K}$, at relative humidity $36.8 \%$. For water at $20{ }^{\circ} \mathrm{C}\left(25^{\circ} \mathrm{C}\right)$, we have $\lambda_{K}=\frac{\gamma_{L} \Omega}{k_{B} T}=0.54(0.52) \mathrm{nm}[21]$.

Considering the maximum distance between the planes of wetting $h=2 r<2\left|r_{K}\right|$, it is easy to see that the equilibrium meniscus can be formed only between two sections of the surfaces that are apart no more than $2\left|r_{K}\right|$.
The dependence of $2 r_{K}$ on the relative humidity is presented at Table 1.

Table 1. The limit initial height of meniscus in dependence on relative humidity.

\begin{tabular}{|c|c|c|c|c|}
\hline$p / p_{s} \%$ & 30 & 50 & 70 & 80 \\
\hline $2\left|r_{K}\right|, \mathrm{nm}$ & 0.90 & 1.56 & 3.03 & 4.84 \\
\hline
\end{tabular}

\section{Force meniscus characteristics}

In the meniscus formation, the attraction force appears due to two reasons $[22,23]$ :

(1) The direct liquid surface tension action $F_{s t}$ pulls the tip and the surface to each other around the meniscus perimeter.

(2) The curved liquid surface is the cause of Laplace pressure, being negative with respect to external pressure.

Our task is to choose the most effective approaches:

(a) to calculate the pull-off force of the probe the given radius from the plane surface,

(b) to describe the "force-distance" dependence $F_{\text {tot }}(s)$ until this job is in the interval $s \in\left(0, s_{\max }\right)$, as well as

(c) to use these values for the local surface diagnostics.

From Fig. 3:

$F_{S t}=2 \pi a \gamma_{L}$,

where $\gamma_{L}=73 \mathrm{mN} / \mathrm{m}$ is water surface tension at $20{ }^{\circ} \mathrm{C}$ $\Delta P_{\text {cap }}=\gamma_{L}(1 / a-1 / r)$.

A suction effect arises as a result of Laplace pressure, it compresses the wetted surfaces to each other with the force $F_{\text {lap }}$. The suction effect is calculated as the product of Laplace pressure with the meniscus cross section

$F_{\text {lap }}=\pi a^{2} \Delta P_{\text {cap }}$.

The total vertical component of the force in each section of the meniscus is constant:

$F_{\text {tot }}=F_{\text {lap }}+F_{\text {st }}$.

Let us estimate the forces for the two nanomeniscus geometries (Table 2). They differ in the "probe-surface" length $s$, meniscus height $h$ and meridional radius magnitude $r$. In both cases, the azimuthal radius is approximately the same $a \approx 10 \mathrm{~nm}$ (Fig. 3).

Obviously, the absolute value of Laplace pressure is much higher (in the nanoscale) than the atmospheric

Table 2. Forces for two nanomeniscus geometries.

\begin{tabular}{|c|c|c|c|c|c|c|c|}
\hline$s, \mathrm{~nm}$ & $a, \mathrm{~nm}$ & $h, \mathrm{~nm}$ & $|r|, \mathrm{nm}$ & $\Delta P_{c a p}, \mathrm{~atm}$ & $F_{s t}, \mathrm{nN}$ & $F_{\text {lap }}, \mathrm{nN}$ & $F_{\text {tot }}, \mathrm{nN}$, \\
\hline 1.0 & 9.965 & 2.093 & 1.300 & -482 & 4.57 & 15.23 & 19.8 \\
\hline 0.0 & 10.17 & 1.093 & 0.679 & -1000 & 4.67 & 32.61 & 37.27 \\
\hline
\end{tabular}


pressure. Therefore, it is not interpreted as a difference of pressures inside and outside the meniscus, but as the internal tensile pressure in the fluid, which pull apart the water molecules from each other. It is also evident that the surface tension force is about 12 to $20 \%$ of the total value $F_{t o t}$, and cannot be considered as the small one. In the approximate models, one tries to avoid this additive force and uses instead of the neck of meniscus as a calculating section, one of its broader bases, where vertical component $F_{s t}$, is small. Therefore, typical values of pull-off forces are about several tens of $\mathrm{nN}$, which are 10 times greater than the van der Waals forces.

Substituting (4), (5), (6) into (7), we obtain:

$F_{\text {tot }}=2 \pi a \gamma_{L}-\pi a^{2} \Delta P_{c a p}$.

We introduce the average cosine $\varsigma=\frac{1}{2}[\cos (\Phi)+\cos (\Theta)]$. Two of curvature radii can be expressed through the angle $\varphi$ (Fig. 1):

$h=2 r \zeta \quad$ and $h=R(1-\cos \varphi)+s \quad(h-$ meniscus total height). obtain:

Equating the two expressions for the height, we

$r=\frac{R(1-\cos \varphi)+s}{2 \varsigma}$.

From Fig. 3 we have:

$a=b-\delta=R \sin \varphi-r[1-\sin (\theta+\varphi)]$.

The substitution of these values into (7) gives the desired force value. However, both radii depend on the unknown filling angle and from each other as well. To resolve this problem, the third equation, Kelvin relation (3) is usually used. Equation (3) allows to calculate the average meniscus surface curvature at a given humidity at equilibrium (and estimate $\Delta P_{\text {cap }}$ ), but it does not allow to determine both radii separately. As a result, equations (3), (9), and (10) give a closed system of 3 nonlinear equations with respect to $r, a$, and $\varphi$ at a given humidity, temperature, radius of the probe, wetting angles, and distance to the surface. Here, it is necessary to make four remarks:

1. Expressions (3), (9), (10) let us directly calculate the pull-off force, or the maximum attraction force $\left.F(s)\right|_{s=0}$, in the wide tip approximation and a small filling angle $1-\cos \varphi \approx \frac{1}{2} \sin ^{2} \varphi$

$$
\begin{aligned}
& F(0) \approx \pi a^{2} \Delta P_{c a p} \approx \pi \gamma_{L} \frac{a^{2}}{r}= \\
& =\pi \gamma_{L} \frac{R^{2} \cdot \sin ^{2} \varphi}{\frac{1}{2} R \cdot \sin ^{2} \varphi} 2 \varsigma=4 \pi \gamma_{L} \cdot R \cdot \varsigma .
\end{aligned}
$$

It is evident that, since the meniscus section area and the meridional curvature radius $r$ are individually proportional to $\sin ^{2} \varphi$, i.e. behave identically with respect to the humidity - the meniscus rupture maximum force does not depend on humidity in the first approximation. It remains only a weak dependence of meniscus rupture maximum force on humidity due to the change in average cosine $\zeta$. This result for a spherical probe is also valid in more accurate theories.

2. The system of equations (3), (9), (10) by mutual substitutions is reduced to a nonlinear equation depending on $\varphi, G(\varphi)=0$, which must be solved numerically for each system configuration, having probe motion from the surface. Respective curves for filling angle on humidity at full tip-sample contact are shown in Fig. 4.

3. Although the equation $G(\varphi)=0$ is strongly nonlinear, its numerical solution allows us to calculate the force curve $F(s)$ for any value of humidity and any relations between the radii of the meniscus and the probe. To determine the unknown sample wetting angle $\Phi$, enough to measure pull-off force. However, it is necessary to use several different probes. This issue will be discussed below in the appropriate section.

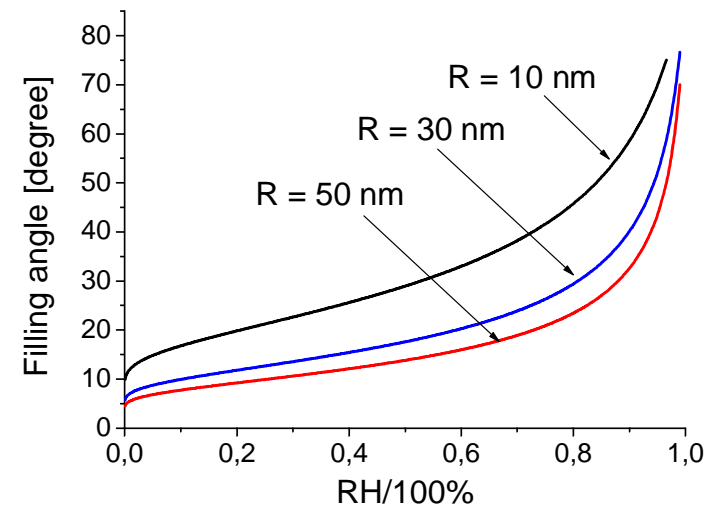

Fig. 4. The dependence of the angle $\varphi$ on humidity at $s=0$ for different tip radii.

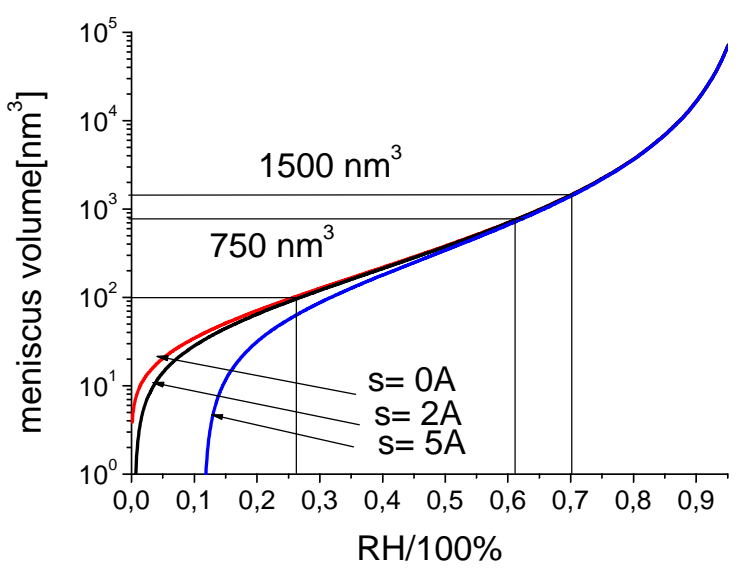

Fig. 5. Meniscus volume $V$ on relative humidity at different tip-sample separations at $R=50 \mathrm{~nm}$.

\section{(C) 2010, V. Lashkaryov Institute of Semiconductor Physics, National Academy of Sciences of Ukraine}


4. There are many published works (one of them is [24]), where the filling angle $\varphi$ is considered as an independent free parameter, being equivalent to relative humidity $p / p_{s}$. Thus, there are models of dependences $F_{\text {tot }}(\varphi)$ or $F_{\text {tot }}(b)$, where $b=R \cdot \sin \varphi$ is the probe wetting area radius. This approach does not inspire confidence, since the filling angle varies with probes detouching from the surface. Its exact value depends on all parameters mentioned above, including the probe radius, at a given humidity (Fig. 4 and 5). In addition, the contact angle behaves differently depending on the measurement conditions: at a constant meniscus volume, or in equilibrium with meniscus constant average curvature. In the first case (3) is accounted only for the initial configuration at $s=0$ (a probe contacts with the surface), then (3) is replaced by the equation $d V=0$, where meniscus volume (Fig. 5) can be calculated by formula (2).

Below, we consider a number of approximate models for calculating the force curve in an explicit form. These models do not require a solution to equations nonlinear in $\varphi$.

\section{Direct pull-off forces and force curves using simplified models [22, 23]}

Below we assume that the filling angle $\varphi$ is small. This is an approximation of low humidity $(\leq 30 \%)$. Hence, $\theta \approx \Theta$ and the angle $\Theta$ is treated as the probe material wetting angle, and $\zeta$ is considered as the average cosine of two materials wetting angle.

\section{Force curves at the meniscus constant curvature mode}

In simplified models it is easier to calculate the force not in meniscus neck section, but in the section of radius $b$, where $F_{s t}$ has its vertical component that is proportional to $\sin \Theta$

$$
\begin{aligned}
F_{\text {tot }}= & \left.2 \pi \gamma_{L} b \cdot \sin \Theta+\pi b^{2} \cdot \gamma_{L}(1 / a-1 / r)\right] . \\
& \text { Substituting } b=R \cdot \sin \varphi, \text { we get: } \\
F_{\text {tot }}= & \pi \cdot \gamma_{L} R \cdot \sin \varphi[2 \cdot \sin \Theta+R \cdot \sin \varphi \cdot(1 / a-1 / r)] .
\end{aligned}
$$

We use the approach of "big tip" and "thick meniscus" $R>>a$ and $a>r, s$. This approach allows us to neglect the first term in square brackets (in the case, where the angles $\varphi$ and $\Theta$ are not very large, and $\varphi$ is not very small) and ratio $1 / a$ in round brackets. Then, for the force module we have:

$$
F \approx \pi \cdot \gamma_{L} R^{2} \cdot \sin ^{2} \varphi \cdot \frac{1}{r}
$$

To calculate $\sin ^{2} \varphi$ we find $\cos \varphi$ from (7), then we use the series expansion $(1-x)^{2} \approx 1-2 x$

$\cos \varphi=1-\frac{2 r \varsigma-s}{R}, \cos ^{2} \varphi=\left(1-\frac{2 r \varsigma-s}{R}\right)^{2} \approx 1-\frac{4 r \varsigma-2 s}{R}$, $\sin ^{2} \varphi=1-\cos ^{2} \varphi=\frac{4 r \varsigma-2 s}{R}$.
Finally we have:

$$
\begin{aligned}
& F \approx \pi \cdot \gamma_{L} R^{2} \cdot \sin ^{2} \varphi \cdot \frac{1}{r}=2 \pi \cdot \gamma_{L} R(2 \varsigma-s / r)= \\
& =2 \pi \cdot \gamma_{L} R(\cos \Theta+\cos \Phi-s / r) .
\end{aligned}
$$

One can see that in the approximate model (13) the force linearly decreases with the distance; its maximum value $(s=0, \Theta=\Phi \approx 0)$ does not exceed $4 \pi \cdot \gamma_{L} R=$ $45.87 \mathrm{nN}$ for the probe of $R=50 \mathrm{~nm}$. The maximum pull-off distance $s=s_{\max }$ does not exceed $2 r$. At equilibrium, from Kelvin relation:

$$
r=-\frac{\lambda_{K}}{\ln \left(p / p_{s}\right)} \text {. }
$$

Moreover, in equilibrium, the radius $r$ is constant (we have neglected by $1 / a$ ). Therefore, the schedule angle of the graph $F=F(s)$ is determined only by the relative humidity value (Figs 6 and 7). Thus, the force curve is not very informative at measurements under equilibrium conditions in the simplified theory, from the sample diagnostic point of view.

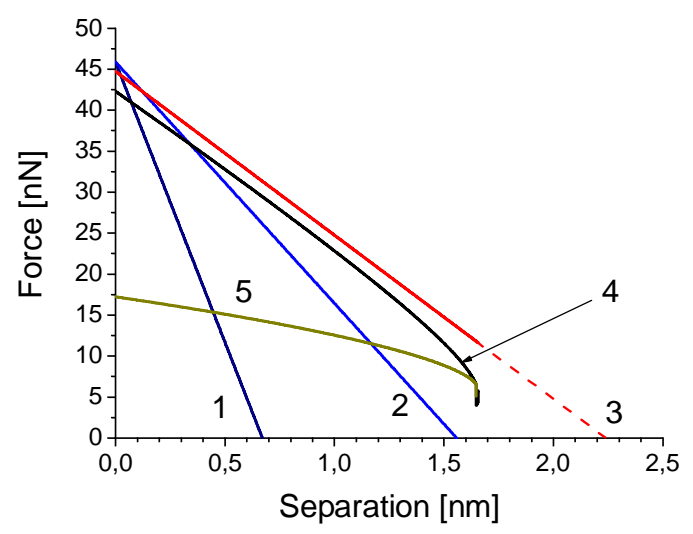

Fig. 6. Force curve at a constant curvature for three relative humidity values $(1-20 \%, 2-50 \%, 3,4-66 \%)$, and filling angle (5). 1,2,3-analytical, 4, 5 - toroidal approximation.

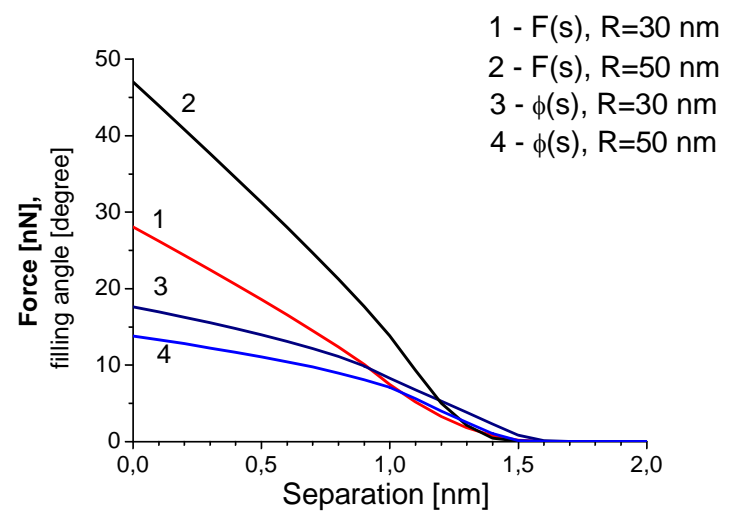

Fig. 7. Force curve and filling angle dependences on separation for equilibrium meniscus at $\mathrm{RH}=50 \%$ for two values of tip radius. 
It can be seen that (if the model is applicable) meniscus-rapture distance values do not exceed a couple of nanometers. However, a more accurate toroidal model (Figs 6,7) gives small deviations from linearity right before the meniscus rupture. To use this fact for diagnostic purposes, it is necessary to increase the accuracy of probe spatial motion in this range.

\section{Force curves at the constant meniscus volume mode}

Let us consider the second version of a simplified theory, when the probe detouches either fast enough, or when the liquid slowly evaporates and condenses, that the equilibrium meniscus with the environment can not be established during the measurement. In this case, the initial meniscus volume is fixed, and the curvature radius varies with the probe motion.

To calculate the liquid volume in the meniscus, we approximate the pendular ring shape with a cylinder having the height $h=R(1-\cos \varphi)+s$ with the radius $b=R \cdot \sin \varphi$, and subtract from it the volume wet probe cap value $\frac{\pi}{6} H \cdot\left(3 b^{2}+H^{2}\right)$, where $H=R(1-\cos \varphi)$ is the cap height. After all the substitutions and simplifications in the small angle approximation $\varphi$, when $1-\cos \varphi \approx \frac{1}{2} \sin ^{2} \varphi$, we get

$V=\frac{\pi}{2} R^{2} \sin ^{2} \varphi \cdot\left(2 s+\frac{R}{2} \sin ^{2} \varphi\right)$.

Substituting $\sin ^{2} \varphi=\frac{4 R \varsigma-2 s}{R}$, we obtain

$V=\pi R\left(4 r^{2} \varsigma^{2}-s^{2}\right)$ or

$r^{2}=\left(\frac{V}{\pi R}+s^{2}\right) / 4 \varsigma^{2}$ and $r=\sqrt{\left(\frac{V}{\pi R}+s^{2}\right)} / 2 \varsigma$.

Substituting $r$ into (13), we obtain the final formula for the force curve (Fig. 8a)

$$
\begin{aligned}
& F \approx 4 \varsigma \pi \cdot \gamma_{L} R\left(1-s / \sqrt{\left(\frac{V}{\pi R}+s^{2}\right)}\right)= \\
& =2 \pi \cdot \gamma_{L} R \cdot(\cos \Theta+\cos \Phi)\left(1-\frac{s}{\sqrt{\left(\frac{V}{\pi R}+s^{2}\right)}}\right) .
\end{aligned}
$$

Here, the pull-off force is the same as in the previous cases (11) and (13). The dependence $F(s)$ does not vanish, no matter what the distance is. This conclusion is not valid, however and caused by cylindrical meniscus geometry assumed in the simplified model. In the toroidal approximation (Fig. 8b) the constant volume liquid bridge is ruptured at a finite distance (from 5 to $10 \mathrm{~nm}$ ), due to collapse of concave walls at the neck. Another reason for the rapture is the meniscus equilibration at some moment. After this the constant volume approximation is not valid, and result depends on the probe speed - the probe moves slowly, the earlier meniscus is torn.

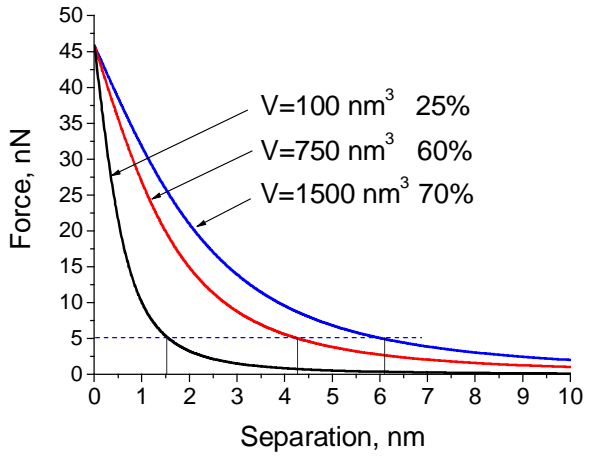

Fig. 8a. Analytical force curves at a constant meniscus volume for three different values of $\mathrm{RH}(R=50 \mathrm{~nm})$.

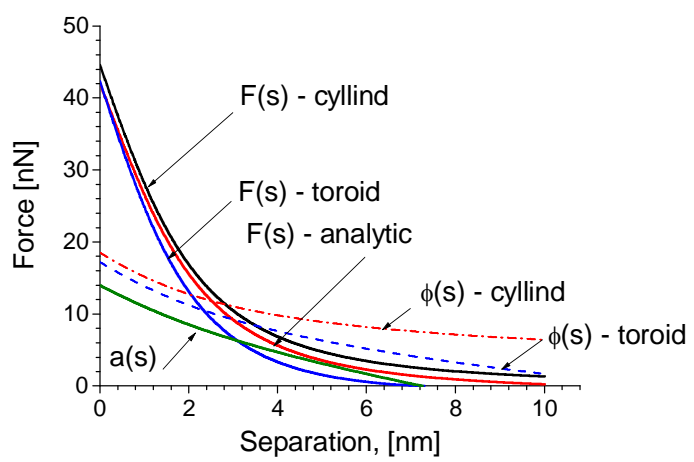

Fig. 8b. Force curves at a constant volume for different models, dependences of filling angle and the neck radius on separation.

It is evident that the force curve (at the initial part $\left.2 r \varsigma=\sqrt{\frac{V}{\pi R}}=-\frac{F(0)}{F^{\prime}(0)}\right)$ contains information about the initial meniscus volume $V=4 \pi R r^{2} \varsigma^{2}$ (Fig. 8a) and allows one to calculate the meniscus curvature initial radius. It enables to control processes in surface nanochemistry and nanolithography.

\section{Energy approach [21]}

In the energy approach, the interaction force is determined from the ratio $F=-\frac{\partial W}{\partial s}$, where $W(s)$ is the total system free energy as a function of the vertical distance and other parameters. If the expression for the energy is written quite fully, considering all the components, we can get more accurate expression for the force, as well as to clarify the nature of the simplifications used in the force approach.

By approximating the meniscus cylinder, let us express the energy as a sum of contributions from different surface parts

$W=W_{1}-W_{0}+W_{2}$,

where

$W_{1}=\pi b^{2} \gamma_{S L}^{S}+2 \pi R H \gamma_{S L}^{Z}=\pi R^{2} \sin ^{2} \varphi\left(\gamma_{S L}^{S}+\gamma_{S L}^{Z}\right)$ 
- two meniscus bases energy (interfaces "liquid-solid"), and

$W_{0}=\pi R^{2} \sin ^{2} \varphi\left(\gamma_{S V}^{S}+\gamma_{S V}^{Z}\right)$

- energy of the same parts of the surface of the sample and probe (in contact with air) before wetting. Here, the superscripts $Z$ and $S$ correspond to the tip and sample, respectively. Summand

$W_{2}=2 \pi b(H+s) \gamma_{L}=W_{3}+A_{s t}=$

$=\pi R^{2} \sin ^{3} \varphi \gamma_{L}+2 \pi b s \gamma_{L}$

corresponds to the total energy of meniscus lateral surface. The first component $W_{3}$ is of higher smallness order $\left(\sin ^{3} \varphi<<\sin ^{2} \varphi\right)$ in comparison with $W_{1}$ and $W_{0}$, the second component $A_{s t}$ corresponds to the liquid lateral surface tension forces work at the meniscus stretching during probe pull-off. Thus, in the small filling angles approximation $(\sin \varphi \approx \varphi \quad$ and $\left.1-\cos \varphi=\frac{1}{2} \sin ^{2} \varphi \approx \varphi^{2} / 2\right)$ there is $W \approx W_{1}-W_{0}$.

Considering that the contact sample and probe angles are determined by the Young relation $\gamma_{L} \cdot \cos \Theta=\left(\gamma_{S V}-\gamma_{S L}\right)$, we obtain

$W=-\pi R^{2} \varphi^{2} \gamma_{L}(\cos \Theta+\cos \Phi)$, from where

$F=-\frac{d W}{d s}=2 \pi R^{2} \gamma_{L}(\cos \Theta+\cos \Phi)\left(\frac{d \varphi}{d s}\right) \varphi$.

The formula for the force contains an unknown factor $\left(\frac{d \varphi}{d s}\right) \varphi$. To define it, let us consider the constant volume approximation. For the liquid volume in the meniscus (meniscus $=$ cylinder - a spherical cap), we have

$$
\begin{aligned}
& V=\pi R^{2} \sin ^{2} \varphi(H+s)-\frac{\pi H^{2}}{3}(3 R-H)= \\
& =\pi R^{2} \sin ^{2} \varphi(H+s)-\frac{\pi R^{3}}{3}(1-\cos \varphi)^{2}(2+\cos \varphi),
\end{aligned}
$$

or after transformation to small $\varphi$

$H \approx R \varphi^{2} / 2 ; \quad(2+\cos \varphi) \approx\left(3-\varphi^{2} / 2\right) ;$

$(1-\cos \varphi)^{2} \approx \varphi^{4} / 4$;

$V \approx \frac{\pi R^{3} \varphi^{6}}{24}+\frac{\pi R^{3} \varphi^{4}}{4}+\pi R^{2} \cdot s \cdot \varphi^{2}$

$V=V(s, \varphi) \approx \pi R^{2} \varphi^{2} s+\pi R^{3} \varphi^{4} / 4$.

In constant volume conditions, all the increments in volume (due to changes in different variables) compensate each other:

$d V=\left(\frac{\partial V}{\partial s}\right)_{\varphi} d s+\left(\frac{\partial V}{\partial \varphi}\right)_{S} d \varphi=0$

Due to $\frac{\partial V}{\partial s}=\pi R^{2} \varphi^{2}$ and $\frac{\partial V}{\partial \varphi}=2 \pi R^{2} \cdot \varphi \cdot s+\pi R^{3} \cdot \varphi^{3}$, it is possible to obtain

$$
\begin{aligned}
& d \varphi / d s=-\frac{V_{s}^{\prime}}{V_{\varphi}^{\prime}}=-\frac{\varphi}{R \varphi^{2}+2 s} \Rightarrow \\
& \Rightarrow \varphi \frac{d \varphi}{d s}=\frac{1}{R} \cdot \frac{1}{1+2 s / R \varphi^{2}} .
\end{aligned}
$$

Substituting this ratio into the expression for the force (19), we finally obtain (formula of Izraelishvili)

$$
\begin{aligned}
& F=2 \pi R \gamma_{L}(\cos \Theta+\cos \Phi)\left(\frac{1}{1+2 s / R \varphi^{2}}\right)= \\
& =\frac{2 \pi R \gamma_{L}(\cos \Theta+\cos \Phi)}{1+s / H}
\end{aligned}
$$

Since $H=R(1-\cos \varphi) \approx \varphi^{2} R / 2$, this formula, however, is not closed and is not suitable for the force curve calculation. In fact, at a constant volume, $H$ depends on the distance $s \Rightarrow H \approx \sqrt{\left(\frac{V}{\pi R}+s^{2}\right)}$. However, (22) gives the correct expression for the pull-off force, which is identical to (11) and (13).

Energy approach shows that, in the final expression for the force, the appearance of contact angles is due to interface energy "solid-liquid", for each of the contacting bodies, and not directly related to real meniscus geometry. Neglecting the meniscus lateral surface energy is equivalent to the neglecting the surface tension forces $F_{s t}$ in the force approach.

\section{Numerical simulation methods for meniscus geometry [25]}

Despite toroidal model is a rather plausible one, it is also one of simplified approaches, when lateral surface is the arc of fixed circle radius $r$, and the horizontal meniscus sections are the circles of variable radius. The average toroid curvature slightly varies along the axis from $1 / r_{a}=1 / r_{K}=(1 / a-1 / r)$ in the neck to $1 / r_{c}=(1 / c-1 / r)$ in the sample surface.

However, in equilibrium meniscus curvature must be constant in every section, and it is inverse Kelvin radius $1 / r_{K}=-\lambda_{K}^{-1} \ln \left(p / p_{s}\right)$. It means that the radii $r=r(y)$ and $a=a(y)$ must be agreed to change along the rotation axis $0 \mathrm{Y}$. The figure, having such a property, is called as nodoid [26]. Let us place origin of coordinates at sample surface plane on meniscus symmetry axis (Fig. 9). Lateral surface profile is calculated as a sequence of small linear elements, whose direction is obtained using the following algorithm:

1) The first linear element starts from some spherical surface point $\left(y_{0}, z_{0}\right)$ in such a way that the angle between this element and the tangent to the sphere gives material sphere wetting angle $\theta$. It is convenient to set the initial filling angle $\varphi=\varphi_{0}$ as an auxiliary parameter. Then

$y_{0}=s+R\left(1-\cos \varphi_{0}\right), z_{0}=R \cdot \sin \varphi_{0}$, and 


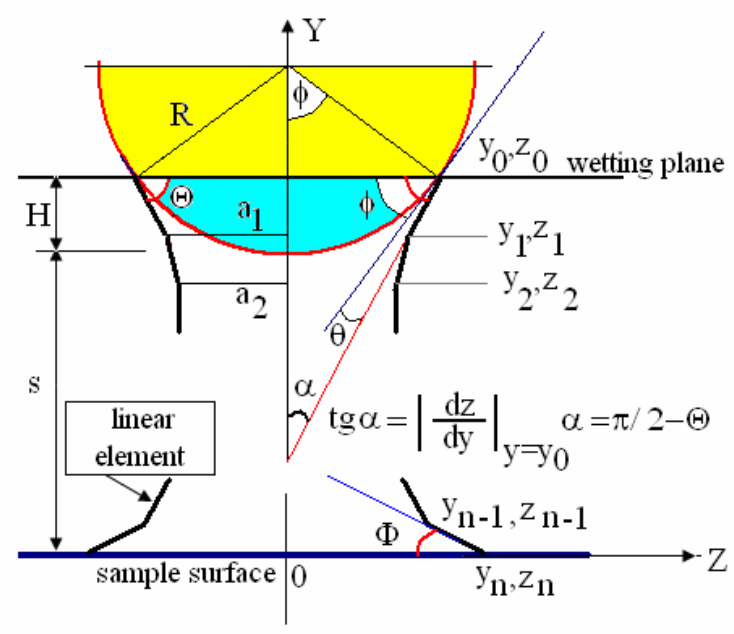

Fig. 9. Numerical simulation illustration of the meniscus profile $z=z(y)$.

$$
\left(\frac{d z}{d y}\right)_{y=y_{0}}=\tan \left(\frac{\pi}{2}-\varphi_{0}-\theta\right) .
$$

2) The endpoint position of the first element $y_{1}, z_{1}$ determines the value of a variable azimuthal radius $a\left(y_{1}\right)=z_{1}$.

3) From $\frac{1}{r_{K}}=-\frac{\ln \left(p / p_{s}\right)}{\lambda_{K}}$ and obtained $a$, we get the second (meridian) radius at the point $r=r\left(y_{1}\right)$, using the formula $+\frac{1}{r}=\frac{1}{a}-\frac{1}{r_{K}}$.

4) The following linear element should be placed to satisfy the resulting curvature meridional radius value $r$

$r=\frac{\left[1+(d z / d y)^{2}\right]^{3 / 2}}{d^{2} z / d y^{2}}$.

5) Since (as the solution of the second order differential equation, or two first order differential equations)

$$
\begin{gathered}
d^{2} z / d y^{2}=\frac{\left[1+(d z / d y)^{2}\right]^{3 / 2}}{r} \\
\Rightarrow\left\{\begin{array}{l}
\frac{d z}{d y}=U \\
\frac{d U}{d y}=\frac{\left[1+U^{2}\right]^{3 / 2}}{r}
\end{array}\right.
\end{gathered}
$$

it is obtained the following linear element endpoint $\left(y_{2}, z_{2}\right)$, where $y_{2}=y_{1}+d y$ and $z_{2}=z_{1}+d z$.

6) The linear element placement process continues till the moment when the profile touches sample surface $y=0$. Thus, it allows to calculate the angle $\Phi^{\prime}$ between the last element and the surface
$\Phi^{\prime}=\frac{\pi}{2}-\arctan (d z / d y)_{y=0}$

If it does not coincide with a given wetting angle, the procedure is repeated from a new starting point (with the new auxiliary angle value $\left.\varphi=\varphi_{1}\right)$ until the desired angle $\Phi$ value is reached. It is clear, if $\Phi^{\prime}>\Phi$, then at the next iteration $\varphi$ should be increased, starting with the more distant probe point from the sample .

From the mathematical point of view, this process corresponds to the boundary problem solution by shooting method. There is one of the borders called as probe wetting plane is mobile. Here, the function and its derivative are given. The second border is sample plane, where only the function derivative is fixed.

Thus, the calculation is carried out at a given humidity, probe radius, and the distance to the surface. Filling angle is automatically excluded from the model parameters.

Meniscus rupture is fixed, if the profile cannot touch the surface at a given angle no matter what $\varphi \in(0, \pi / 2)$. Force calculation is based on formulas similar to (8) and (9).

It is interesting case when the sample or the probe is separately hydrophobic. For example, one of cosines is negative. Simplified formulas (11) and (12) give the ultimate value of the attractive force, whereas the procedure for constructing the meniscus may thus fail (because the meniscus can not have inflection points where profile curvature changes sign). The latter is equivalent to the absence of a bridge between the tip and surface and the attraction force is of zero value.

Let us list briefly the main results obtained in the framework of this approach [25] applicable to a spherical probe:

- For thick probes with radius $R \geq 100 \mathrm{~nm}$, the pull-off force is weakly dependent on humidity. Even for $R=15 \mathrm{~nm}$, it decreases by $20 \%$ when changing $\mathrm{RH}$ from 10 to $50 \%$.

- For a finite distance from the sample, the force considerably varies (increasing by several times) with humidity increasing, especially in the range of $10-50 \%$. The stretched meniscus actively absorbs the moisture from the environment.

- The comparison of calculations in the framework of this approach and the toroidal approximation gives discrepancy no more than 1-3\%. The discrepancy increases with decreasing $R$ and increasing humidity.

- In exact approach, the force is also proportional to the contact angle cosine, as in the approximate models, for both large and small $R$ values.

- The force curves calculated at constant curvature and constant volume are qualitatively similar to curves (13) and (14), calculated from the corresponding approximate models (Figs 6-8). 
- The dependence of force on the humidity is very sensitive to the probe shape, especially not in contact, but at finite distances from the surface.

In general, this approach is not more complicated or time-consuming than the toroidal approximation and can be used in practice, along with other approaches.

\section{Amendments to adhesion forces}

All the arguments above are related only to the capillary forces induced by liquid in between two solids. An additional factor is the adhesion force due to direct contact between two different solids inside the liquid meniscus.

Let us use the Derjaguin approximation [21], which relates the interaction force $F_{a d}$ between curved objects of radii $R_{1}$ and $R_{2}$, and the interaction energy between flat surfaces (unit area) at the same distance $s$

$F_{a d}(s)=2 \pi \frac{R_{1} \cdot R_{2}}{R_{1}+R_{2}} W(s)$.

Repeating the arguments [21] for the case, we find that in the case of contact $(s=\sigma)$, in the liquid two single planes system energy is equal to their interface energies sum $W=\gamma_{S L}^{Z}+\gamma_{S L}^{S}$. Hence, the adhesion force between plane and sphere $\left(R_{1}=R\right.$ and $\left.R_{2}=\infty\right)$ :

$F_{a d}=4 \pi R\left\langle\gamma_{S L}\right\rangle$,

where $\left\langle\gamma_{S L}\right\rangle=\frac{1}{2}\left(\gamma_{S L}^{Z}+\gamma_{S L}^{S}\right)$ is the average interface energy of the contacting materials in the given liquid. This formula is valid for indeformable objects. More general case differs from (27) only by a numerical factor [21].

In most cases, the direct adhesion force $F_{a d}$ is low and all the formulas, presented above, remain valid. In addition, this force can be measured directly in a liquid that has been described in this paper. It should also be noted that by measuring one sample in the liquid using two different probes, after an additional third measurement of the probe on the probe (in liquid) you can get all three interface energies values, relating to the sample $\gamma_{S L}^{S}$ and two probes $\gamma_{S L}^{Z_{1}}$ and $\gamma_{S L}^{Z_{2}}$.

\section{Results and discussion}

Typical force curves for 50-nm thick gold film deposited on a glass substrate are shown in Fig. 10. Comparison of force curves obtained in air and in liquid shows that the value of capillary force is by order of magnitude more than that of adhesive forces holding the AFM tip near the sample surface (curves 2). Bridge rupture is happened after stretching for about $5 \mathrm{~nm}$ at the available force $11.8 \mathrm{nN}$ (Fig. 10a, curve 2). Accordingly, the contact with surface in liquid is destructed when tip reaches a distance close to $2 \mathrm{~nm}$ and the force of $1.3 \mathrm{nN}$ (Fig. 10b, curve 2), and there is no significant jump. It is interesting fact, that snap-in part of approaching curve (Fig. 10b, curve 1), where, in contrast to measurements in air, AFM tip is attracted to surface starting from a distance close to $20 \mathrm{~nm}$. Probably, this behavior in the liquid snap-in curve is associated with the uncontrolled surface layer specifics of adsorbed active chemical groups (eg $\mathrm{CH})$, in which liquid is actively manifested.

Relief surface and surface charge (potential) features are illustrated by the thick (several microns) film example of pure polytetrafluoroethylene (PTFE, teflon) and PTFE, filled with multiwalled carbon nanotubes (Fig. 11). Investigated pure PTFE surface has a root-mean-square roughness (RMS) of $3 \mathrm{~nm}$ with the typical curvature radius of single inequalities of $1000 \mathrm{~nm}$ (Fig. 11a). Carbon nanotubes added to the PTFE cause increasing the RMS value up to $4 \mathrm{~nm}$ (Fig. 11c). Characteristic surface features curvature radius is less than tip apex radius and, according to AFM measurements in the tapping-mode, is 10 to $30 \mathrm{~nm}$. For such surfaces, characteristic pull-off force values in the liquid and air are, respectively, 3.7 and $10.7 \mathrm{nN}$ in the case of pure PTFE as well as 0.9 and $8.3 \mathrm{nN}$ for PTFE with CNT (Fig. 11b, d). Thus, there is a clear tendency to reduce the value of capillary forces over rough surfaces where multiple capillary bridges could be formed and their geometry could be distorted due to high local surface curvature in accordance with the Derjaguin's formula.
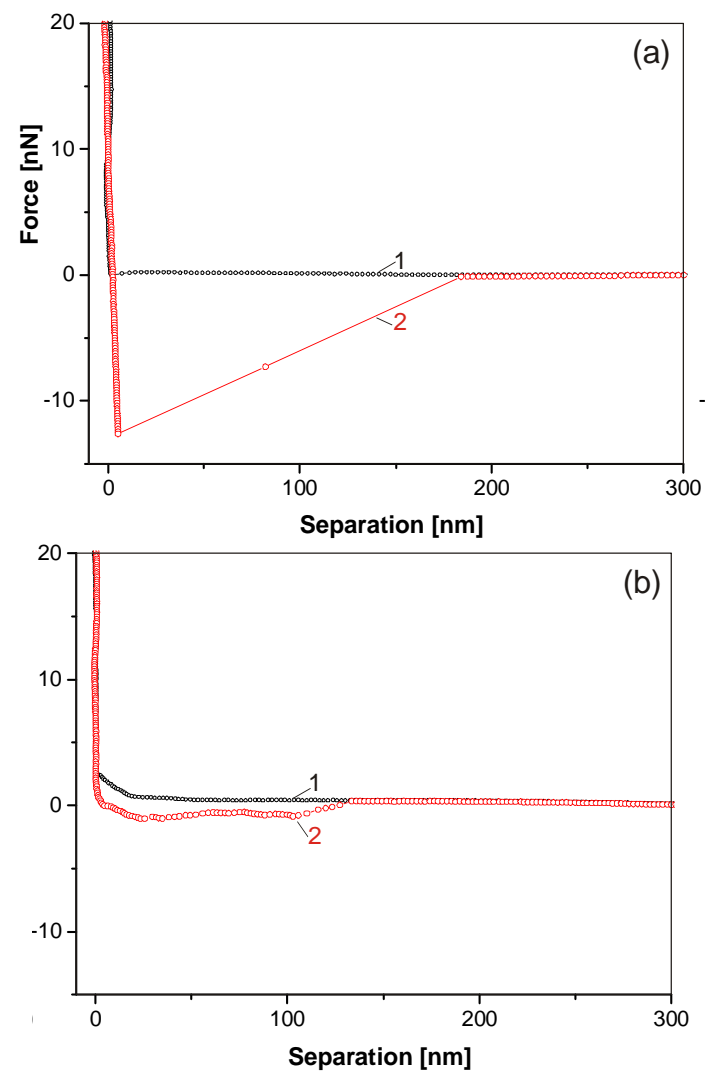

Fig. 10. Force curves for a gold film in the air (a) and liquid (b): approaching curve (1) and withdrawing curve (2). 

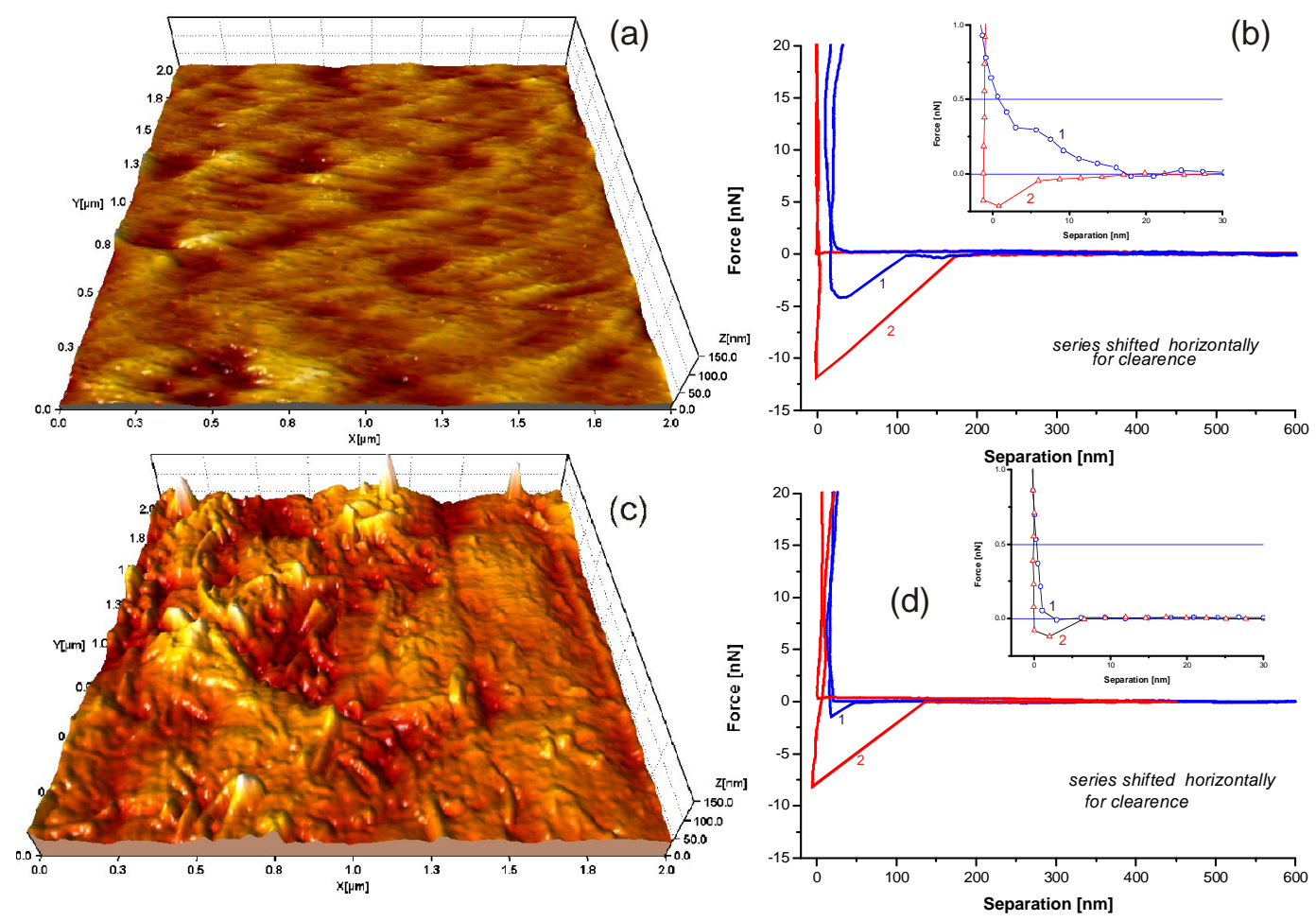

Fig. 11. AFM images surfaces and the corresponding force curves for PTFE (a, b) and PTFE filled with carbon nanotubes (c, d). Curves taken in the liquid are of blue colour (1), in the air are of red colour (2). Inserts in b and d show the snap-in areas of relevant curves.

A characteristic pure PTFE feature is static electric charge presence on the surface, while in PTFE with carbon nanotubes it is practically absent. Such a charge can significantly affect the force curves, especially in liquid. So, in the snap-in curve for pure PTFE in liquid there is no abrupt jump to contact part (see insert in Fig. 11b, curve 1). There is the so called double layer electrostatic repulsion, which starts from the tip-surface distance of $\sim 20 \mathrm{~nm}$. While for PTFE with carbon nanotubes in liquid (insert in Fig. 11d) jump to contact occurs at a distance of $\sim 2.5 \mathrm{~nm}$. This behavior is explained by facilitating charge flow from the surface with conductive carbon nanotubes.

Fig. 12 illustrates the dependence of capillary bridge geometry on the AFM tip withdraw speed. Measurements performed on the artificial diamonds surface, which excludes the possible deformation effects in tip-surface contact vicinity. It is seen, the higher a tip withdraw speed the larger height of bridge: increasing the speed from 20 to $200 \mathrm{~nm} / \mathrm{s}$ causes an increase of the bridge height from 2-5 to 6-10 nm (Figs 12a, b). At the speed of $2000 \mathrm{~nm} / \mathrm{s}$, the bridge is ruptured at the height of 12-15 nm only (Fig. 12c). This behavior qualitatively agrees well with the simulation results presented in Figs 6-8. The slow tip movement corresponds to meniscus constant curvature, a quasi-linear force curve and shorter capillary bridges (Fig. 6). The constant volume mode is realized during fast AFM tip withdrawing (Fig. 8).
It should be noted that shown variations of the tip speed do not significantly affect the derived capillary pull-off force value (Fig. 12, histograms) which is in good agreement with theoretical models discussed above. There is only a slight distribution broadening. Anyway, it is necessary to notice the difference between the measured and the envisaged capillary bridge heights.

Dependences of adhesion forces in air and liquid for various materials are shown in Fig. 13. Generally, there is a capillary forces correlation value from the wetting angle investigated surfaces. However, in these measurements it is particularly important to examine surface cleanliness. Not always correlation of capillary and adhesion forces takes place in liquid.

\section{Surface energy mapping using capillary bridge pull-off force}

From the theoretical models, the meniscus rupture force depends on the average contact angle cosine for the sample and the probe, respectively. Therefore, for surface proper diagnostics there must be a probe calibrated to the wetting angle. Alternatively, we can suggest a method of forces rupture bridge measuring by two different probes on the same sample $\left(F_{1}\right.$ and $\left.F_{2}\right)$ with additional capillary forces measurement probe on the probe $F_{12}=F_{21}$. This method immediately gives the calibration of wettability for both probes $\cos \Theta_{1}$ and 
$\cos \Theta_{2}$, as well as the corresponding map of $\cos \Theta$ value for researched sample:

$$
\left\{\begin{array}{l}
\cos \Theta_{1}+\cos \Phi=A \\
\cos \Theta_{2}+\cos \Phi=B \\
\cos \Theta_{1}+\cos \Theta_{2}=C
\end{array}\right.
$$

Here, $\quad A=F_{1} / 2 \pi \gamma_{L} R_{1}, \quad B=F_{2} / 2 \pi \gamma_{L} R_{2}$, $C=F_{12} / 2 \pi \gamma_{L} R_{1(2)}$. This system solution is elementary: $\cos \Phi=(A+B-C) / 2, \quad \cos \Theta_{1}=(A+C-B) / 2$ and $\cos \Theta_{2}=(B+C-A) / 2$.

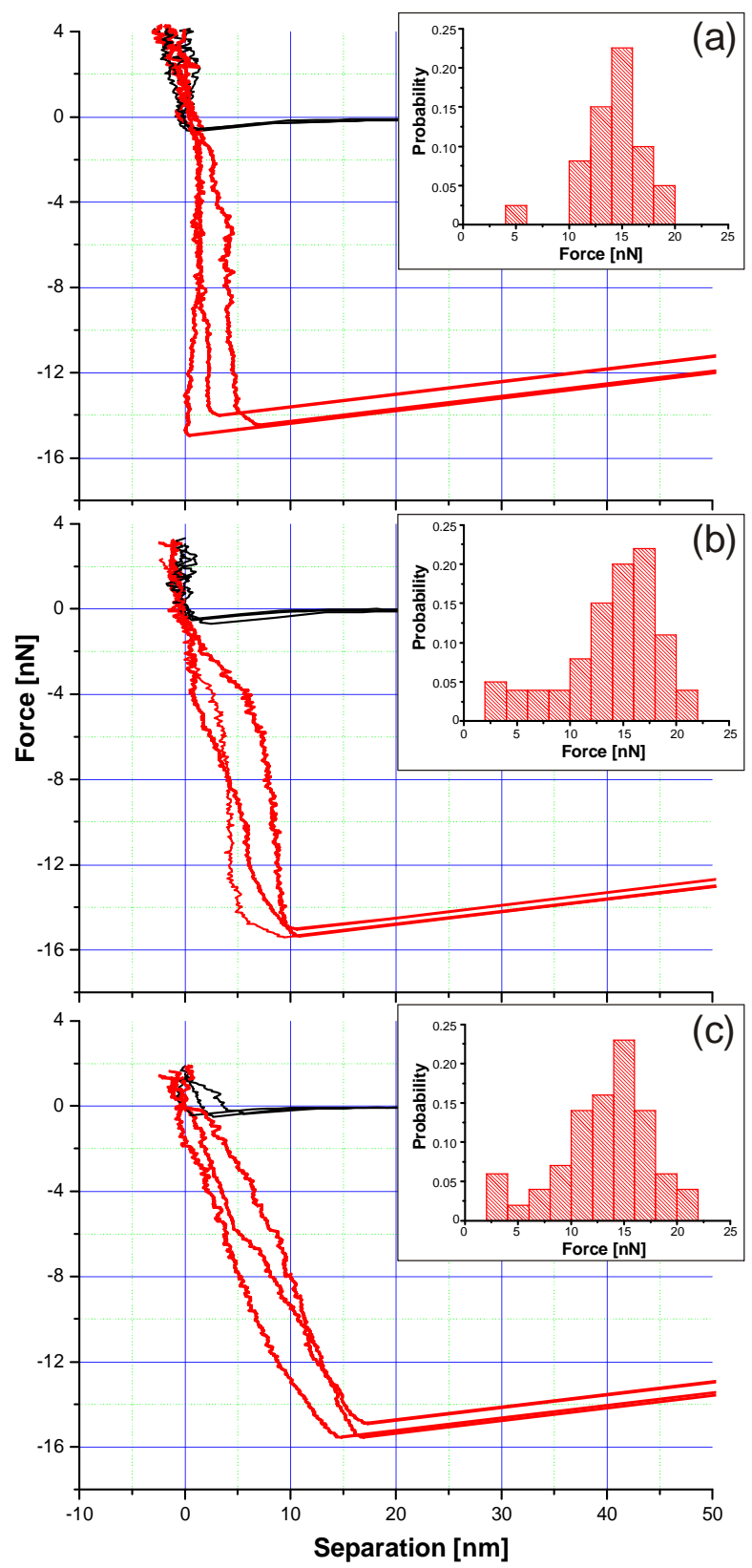

Fig. 12. Force curves and capillary forces histograms (inserts) for the diamond surface depending on capillary bridge forming speed: $20 \mathrm{~nm} / \mathrm{s}$ (a), 200 (b), and 2000 (c).
Likewise, it is possible to make wetting angle intercalibration for three probes at the same time. Having the wetting angle map data for the sample (Young's formula [21])

$$
\cos \Phi=\left(\gamma_{S V}^{S}-\gamma_{S L}^{S}\right) / \gamma_{L}
$$

as well as adhesion forces measurements on the same sample in liquid, giving value $\gamma_{S L}^{S}$, solid surface energy mapping can be realized with nanometer resolution. This very useful result can be a diagnostic tool for established and new technological trends in nanolithography, the local water chemistry, local (electro) chemistry of materials, as well as corrosion processes study and surfactants traces detection on the surface. This method can be used not only for diagnostics but also for the local chemical surface modification due to the reagent injection directly to the meniscus. Moreover, all reactions take place in the liquid under high tensile pressure. It is obvious that control the meniscus geometry and its force characteristics can be used here to monitor process kinetics.

Naturally, during this program performing number of methodological problems increases. To such problems are referred geometric relief treatment (especially in the nanoscale), a partial surface deformation in contact with the probe, etc. However, the good news is that most of these problems can be solved within AFM-spectroscopy framework. Another problem is in the possible restrictions imposed on the applicability of the formulas Young, Derjaguin, and Kelvin [21] for drops and menisci of nanoscale sizes, as well as all given above (in essence, a continuum) models. In particular, it remained unsettled question about the need to incorporate linear tension on the liquid perimeter in contact with solid, as well as considering dependence of the meniscus surface tension on meniscus curvature. Of course, there are required additional researches, including new atomistic model creation. At this stage, we observe good qualitative agreement with continuum models and the experimental results presented here.

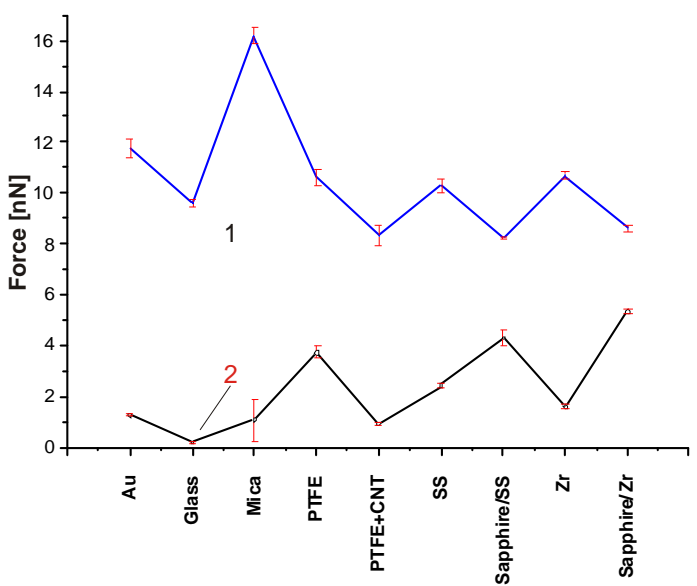

Fig. 13. Capillary bridge rupture value for different materials in air (1) and magnitude of adhesive forces in the liquid for related materials (2). SS - stainless steel. 


\section{Conclusions}

In this paper, authors make an attempt to develop systematic theoretical and experimental approach for application of capillary forces as a unique diagnostic tool for a real surface basing on an atomic force spectroscopy technique. There is comparative analysis of the most successful theoretical approaches and experimental illustration of several effects that define nanocapillary interactions on real surfaces. Introduced there are experimental results of rupture capillary bridge force measurements in air and adhesion forces inside liquid by AFM tip probing a surface. The investigated materials differed in conductivity, topography, adsorbed layer presence etc. It is shown that theoretical models describe quite well the processes and, in particular, bridge rupture dynamics with a vertical tip displacement at different speeds. It is expected that usage of more advanced numerical models, adequately constructed on the base of the simplest theories, allows complex heterogeneous surfaces diagnostics using nanocapillary forces. In addition, an important role is played by proper strategy for the experiment using all force microscopy advantages. The analysis allowed to propose a new approach to diagnose real surface local energy parameters using probes of different properties, at agreed spatially measurements both in air and liquid, using topometric and surface potential data.

\section{References}

1. R. Jones, H.M. Pollock, D. Geldart, A. Verlinden, Inter-particle forces in cohesive powders studied by AFM: effects of relative humidity, particle size and wall adhesion // Powder Technology 132, p. 196210 (2003).

2. Q. Ouyang, K. Ishida, K. Okada, Investigation of micro-adhesion by atomic force microscopy // Appl. Surf. Sci. 169-170, p. 644-648 (2001).

3. Guoxin Xie, Jianning Ding, Beirong Zheng, Wei Xue, Investigation of adhesive and frictional behavior of GeSbTe films with AFM/FFM // Tribology International 42, p. 183-189 (2009).

4. Zoya Leonenko, Eric Finot, Matthias Amrein, Adhesive interaction measured between AFM probe and lung epithelial type II cells // Ultramicroscopy 107, p. 948-953 (2007).

5. S.J. Yuan, S.O. Pehkonen, AFM study of microbial colonization and its deleterious effect on 304 stainless steel by Pseudomonas NCIMB 2021 and Desulfovibrio desulfuricans in simulated seawater // Corrosion Sci. 51, p. 1372-1385 (2009).

6. Seong S. Choi, V.V. Anh, M.S. Joo, Y.C. Kim, K.J. Kim, D.W. Kim, Analysis of force distance curve for biomolecule imaging // Current Appl. Phys. 6S1, p. 247-250 (2006).

7. V. Yanchenko, T. Alekseyeva, V. Bobrov, O. Lazarenko, P. Lytvyn, O. Lytvyn, S. Oshkaderov,
Methode physique de determination de la biocompatibilite des materialix pour les implants intravesculaires // 8 Congres Francophone de cardiologie Interventionnelle, Paris, 11-13 October 2006, p. 114.

8. L. Zitzler, S. Herminghaus, and F. Mugele, Capillary forces in tapping mode atomic force microscopy // Phys. Rev. B 66, 155436-1-155436-8 (2002).

9. Dimitrios Fotiadis, Simon Scheuring, Shirley A. Muller, Andreas Engel and Daniel J. Muller, Imaging and manipulation of biological structures with the AFM // Micron 33(4), p. 385-397 (2002).

10. Werner Frammelsberger, Guenther Benstetter, Janice Kiely, Richard Stamp, C-AFM-based thickness determination of thin and ultra-thin $\mathrm{SiO}_{2}$ films by use of different conductive-coated probe tips // Appl. Surf. Sci. 253, p. 3615-3626 (2007).

11. Midori Kato, Masayoshi Ishibashi, Seiji Heike and Tomihiro Hashizume, Nanofabrication using atomic force microscopy lithography for molecular devices // Jpn. J. Appl. Phys. 41, p. 4916-4918 (2002).

12. P.M. Lytvyn, O.Ya. Olikh, O.S. Lytvyn, O.M. Dyachyns'ka, I.V. Prokopenko, Ultrasonic assisted nanomanipulations with atomic force microscope // Semiconductor Physics, Quantum Electronics \& Optoelectronics, 13(1), p. 36-42 (2010).

13. Hans-Jurgen Butt, Brunero Cappella, Michael Kappl, Force measurements with the atomic force microscope: Technique, interpretation and applications // Surf. Sci. Repts. 59, p. 1-152 (2005).

14. V. Cambel, J. Martaus, J. Soltys, R. Kudela, D. Gregusova, AFM nanooxidation process Technology perspective for mesoscopic structures // Surf. Sci. 601, p. 2717-2723 (2007).

15. Paula Gould, Lithography: rewriting the rules // Materialstoday 6, Issue 5, p. 34-39 (2003).

16. William B. Haines, Studies in the physical properties of soils: II. A note on the cohesion developed by capillary forces in an ideal soil // The Journal of Agricultural Science 15, Issue 04, p. 529-535 (1925).

17. R.A. Fisher, On the capillary forces in an ideal soil; correction of formulae given by W. B. Haines // The Journal of Agricultural Science 16, Issue 03, p. 492-505 (1926)

18. L.S. Dongmo, J.S. Villarrubia, S.N. Jones, T.B. Renegar, M.T. Postek, J.F. Song, Experimental test of blind tip reconstruction for scanning probe microscopy // Ultramicroscopy 85, p. 141-153 (2000).

19. Jeffrey L. Hutter and John Bechhoefer, Calibration of atomic-force microscope tips // Rev. Sci. Instrum. 64 (7), p. 1868-1873 (1993).

20. J. Frankenfield, Volumes and areas of pendular rings with non-zero contact angles // http://www.snowman-jim.org/papers/rings/

21. J. Israelachvili, Intermolecular and Surface Forces. Academic Press, San Diego, CA, 1998. 
22. H.-J. Butt and M. Kappl, Surface and Interfacial Forces. WILEY-VCH Verlag GmbH\&Co, KGaA, Weinheim, 2010.

23. H.-J. Butt and M. Kappl, Normal Capillary Forces // Advances in Colloid and Interface Science 146, p. 48-60 (2009).

24. Li Zhao-Xia, Zhan Li Juan, Yi Hou-Hui, Fang HaiPing, Theoretical study on the capillary force between an atomic force microscope tip and a nanoparticle // Chin. Phys Lett. 24, No 6, p. 22892292 (2007).
25. O.H. Pakarinen, A.S. Foster, M. Paajanen, T. Kalinainen, J. Katainen, I. Makkonen, J. Lahtinen and R.M. Nieminen, Towards an accurate description of the capillary force in nanoparticle-surface interactions // Modelling Simul. Mater. Sci. Eng. 13, p. 1175-1186 (2005).

26. F.M. Orr, L.E. Scriven, P. Rivas, Pendular rings between solids: meniscus properties and capillary force // J. Fluid Mech. 67, p. 723-742 (1975). 